Developmental considerations for assessment and treatment of impulsivity in older adults Melissa Liu, B.S.

Eva Argyriou, M.S.

Melissa A. Cyders, Ph.D.

Department of Psychology, Indiana University Purdue University Indianapolis, 402 N. Blackford St., LD124, Indianapolis, IN 46202

Author note: Corresponding Author, Melissa A. Cyders, mcyders@iu.edu, 402 North Blackford Street, LD124, Indianapolis, 1IN, 46202, (317) 274-6752.

Funding: Preparation of this article was supported in part by R01AA027236, P60AA007611, and the Indiana University Addiction Grand Challenge (Cyders).

Conflict of Interest: All authors declare that they have no conflicts of interest.

This is the author's manuscript of the article published in final edited form as:

Liu, M., Argyriou, E., \& Cyders, M. A. (2020). Developmental Considerations for Assessment and Treatment of Impulsivity in Older Adults. Current Topics in Behavioral Neurosciences, 47. https://doi.org/10.1007/7854_2019_124 


\begin{abstract}
Impulsivity is an important factor in many clinical disorders, especially alcohol and substance use disorders. Most of the research on impulsivity in this domain has focused on adolescence and young adulthood, as this developmental period is characterized by onset of and escalation in alcohol and substance use, likely driven in part by brain development patterns. Although many individuals eventually "mature out" of these behaviors in middle adulthood, a critical subset of people do not. The role of impulsivity in middle-to-older adulthood, when certain individuals transition from normative to disordered substance use, has not been carefully examined. The goal of this paper is to review the literature on measuring and modifying impulsivity from adolescence through older adulthood, with a special focus on middle-to-older adulthood. We propose that impulsivity research should include data on middle-to-older adulthood as an important time of transition to disordered use. We consider how impulsivity might have unique meaning at different stages of the adult lifespan and suggest modifications for assessing and treating impulsivity in older adults.
\end{abstract}

Keywords: impulsivity, substance use, alcohol use, adulthood, age 


\section{Developmental considerations for assessment and treatment of impulsivity in older adults}

There is a vast literature linking impulsivity to a wide range of clinical problems(see Um, Hershberger, Whitt, \& Cyders, 2018), especially alcohol and substance use disorders (e.g., Coskunpinar, Dir, \& Cyders, 2013; Dick et al., 2010; Gerard Moeller \& Dougherty, 2002; Verdejo-García, Lawrence, \& Clark, 2008). Most of this research has focused on adolescence and young adulthood, when impulsive and risky behaviors, such as alcohol and substance use (e.g., Young et al., 2002), are common. This period also corresponds with brain maturation that is thought to contribute to these behaviors (Blakemore \& Robbins, 2012; Casey, Jones, \& Hare, 2008; Crone \& Dahl, 2012). Although many individuals eventually “mature out” of these behaviors after the early 20's (Littlefield \& Sher, 2015; Littlefield, Sher, \& Wood, 2009), a subset of individuals do not and instead transition to problem use during middle adulthood (Heyman, 2013). This later period is clinically important but has received little attention. For reasons that are not fully understood, alcohol and substance use appear to be increasing in middle-to-older adults (Breslow, Castle, Chen, \& Graubard, 2017). The goal of this paper is to review the literature on measuring and modifying impulsivity from adolescence through older adulthood, with a special focus on middle-to-older adulthood. We consider how impulsivity might have different meanings at different stages of the adult lifespan and suggest specific modifications for assessing and treating impulsivity in older adults.

\section{Measurement of Impulsivity}

When researchers discuss and measure impulsivity, they are usually referring to one of two different levels of measurement or analysis, which, by and large, do not highly correspond (see Cyders \& Coskunpinar, 2011). The first is at the level of personality trait, i.e., the stable underlying construct of impulsivity, which commonly utilizes self-report questionnaires to assess 
a wide range of different traits, such as acting without thinking or seeking out exciting experiences (see Evenden, 1999 for a full review on this topic). Because of the varied definitions across studies, Whiteside and Lynam (2001) conducted a factor analysis of existing personalitybased impulsivity scales to identify the common traits assessed across these measures. The result of this analysis was the UPPS-P Impulsive Behavior Scale (Lynam, Smith, Whiteside, \& Cyders, 2006; Whiteside \& Lynam, 2001). Three main domains are included in this scale: deficits in conscientiousness, which comprises not thinking before acting and not following tasks to completion; sensation seeking, which is seeking out new and exciting experiences and sensations; and emotion-based rash action, which comprises acting rashly in response to negative ('negative urgency') and positive ('positive urgency') emotions (see Cyders \& Smith, 2007; Cyders et al., 2007; Lynam et al., 2006; Whiteside \& Lynam, 2001).

The second level of measurement is the behavioral manifestation of the trait, i.e., assessing impulsive behavior, usually via an objective behavioral task. Behavioral measures of impulsivity seek to assess behavioral responding in the moment, and thus are more 'state-like' than 'trait-like' (Cyders \& Coskunpinar, 2011; Sharma, Markon, \& Clark, 2014). Over the years, many behavioral lab tasks have been developed, assessing different aspects of impulsivity. This field also sought to compile and group these measures into representative task domains of impulsivity. Dick and colleagues (2010) categorized these assessments into five domains: prepotent response inhibition (suppressing dominant or automatic responses), resistance to distractor interference (avoiding distraction in goal-directed behavior), resistance to proactive interference (avoiding memory intrusions in goal-directed behavior), delay response (choosing a larger delayed reward over an immediate reward), and distortions in elapsed time (judging 
passing time accurately). Others have categorized these tendencies differently (Aragues, Jurado, Quinto, \& Rubio, 2011; Fineberg et al., 2014; Sharma et al., 2014).

Overall, objective behavioral measures of impulsivity correlate poorly with self-report measures, likely because trait-like and state-like impulsivity are different things (e.g., Cyders \& Coskunpinar, 2011). Importantly though, even the separate constructs within each measurement type are quite independent and predict differential aspects of alcohol and substance use behaviors. For example, the three UPPS-P domains only have small to moderate intercorrelations correlate with each other (Cyders \& Smith, 2007), suggesting they are separate, though somewhat related, tendencies toward impulsive action. Other work has supported these lower correlations among different constructs within personality-based and objective behavioral measurements of impulsivity, showing that disparate constructs differentially relate to substance use outcomes (e.g., Coskunpinar et al., 2013; Dick et al., 2010; Smith et al., 2007). Measuring impulsivity constructs at the sub-facet level leads to more precise measurement and more accurate prediction of substance use outcomes (see Smith et al., 2007; Smith, Fischer, \& Fister, 2003).

A construct that has been formulated as distinct from impulsivity is compulsivity. Compulsivity refers to behaviors that are outside of one's control, difficult to stop, and that have become insensitive to environmental consequences of the behaviors (see Koob \& Le Moal, 2008). George Koob (2004) has proposed that whereas alcohol and substance addiction is driven by impulsivity at the early stages of substance use, it is controlled by compulsivity at the later stages. In this model, one moves from positive reinforcement reasons for substance use (e.g., to feel the high) to negative reinforcement motivations (e.g., to avoid withdrawal symptoms) (Koob, 2004). Thus, this suggests that impulsivity is important for initiation of substance and 
alcohol use, but that compulsivity comes into play in the transition to a substance use disorder. This has been supported by some research where impulsivity is an important factor in substance use in adolescence and young adulthood (see reviews by Stautz \& Cooper, 2013; VanderVeen, Hershberger, \& Cyders, 2016), whereas compulsivity is more important in middle to older adulthood as people transition to disorder-level use (e.g., Koob \& Le Moal, 2008). Yet, these conclusions raise questions about the operational definition of compulsivity, and whether the claim that compulsivity plays a greater role later in the drug use trajectory could be confounded by the samples typically studied in impulsivity research (see a review by Argyriou, Um, Carron, \& Cyders, 2018). If the participants of studies investigating impulsivity are mainly adolescents or young adults, this may give the impression that impulsive behaviors are more important in these younger groups, eventually biasing impulsivity-based theory. The distinction and relative importance of impulsivity vs. compulsivity in the development and maintenance of substance use disorders has recently been questioned. Zorrilla and Koob (2019) proposed that a specific impulsivity trait, namely negative urgency, is the bridge that facilitates the transition from social or normative substance use to addiction. Indeed, meta-analytic findings indicate that negative urgency is the personality-based impulsivity trait that has the highest associations with alcohol dependence (Coskunpinar et al., 2013). Additionally, certain brain mechanisms appear to underlie both negative urgency and addiction (Um, Whitt, Revilla, Hunton, \& Cyders, 2019), further strengthening the link between this trait and addiction. Thus, focusing primarily on adolescents and young adults in studying how impulsivity underlies alcohol and substance use disorder risk may have led to a misunderstanding as to which traits underlie how addiction develops and is maintained. It has been proposed that whereas sensation seeking is important for 
the onset of substance use, negative urgency is more important for the transition to problematic use (Smith, Fister, \& Fischer, 2007).

\section{Developmental Changes in Impulsivity Across the Lifespan}

Impulsivity changes across the lifespan, corresponding with maturational changes in the brain. In general, personality-based impulsivity traits increase with the onset of puberty through the early 20s and then level off in the transition into middle-adulthood (Littlefield et al., 2016; Romer \& Hennessy, 2007; Steinberg et al., 2008), although this pattern is not as robust for sensation seeking (Collado et al., 2014; Harden \& Tucker-Drob, 2011; Pedersen et al., 2012). For behavioral measures, delayed reward discounting decreases from adolescence into adulthood (Green et al., 1994; Olson et al., 2007; Prencipe et al., 2011) and response inhibition performance improves from adolescence into adulthood (Jaeger, 2013; López-Caneda et al., 2014). It has been suggested that the increase in impulsivity during adolescence results from the gap between the early development in the affective processing system (including areas of the mesolimbic dopamine circuit) and later development of the cognitive control system (including the lateral prefrontal cortex and parts of the anterior cingulate cortex) (Ernst and Fudge, 2009; Somerville et al., 2010; Stautz and Cooper, 2013; Steinberg, 2008). This gap in maturation may make it difficult for adolescents to restrain impulses toward rewarding experiences, such as substance use. Another theory highlights the heightened sensitivity of a maturing 'reward system' to social and affective incentives that become more salient due to a highly flexible (as opposed to underdeveloped) cognitive control system (Crone \& Dahl, 2012; Galvan, 2010).

Starting around the age of 25, impulsive and risk-taking behaviors decline, corresponding with reductions in impulsivity traits that level off in middle adulthood -a phenomenon called “maturing out" (Littlefield \& Sher, 2016; Vergés et al., 2012). However, a small but clinically 
relevant percentage of adults increases their substance use and transitions to more severe patterns of use indicative of a substance use disorder. More research is needed to understand how impulsivity may drive this maintenance or increase in problematic substance use in middle adulthood.

Limited research examining impulsivity in middle-to-older adulthood is problematic for research and clinical application. Because impulsivity has not been considered an important determinant of substance use among middle-older adults, it is not known whether impulsivity is simply understudied in this group or whether it is indeed less relevant for middle-to-older adults. If impulsivity plays a lesser role in substance use for older adults, or if their substance use is driven by different forms of impulsivity, this would suggest a need for different strategies for identifying, preventing, and treating this population compared to younger individuals. Applying treatments that work for younger populations to older people may be mis-directed (Argyriou et al., 2018). Next, we discuss developmental considerations for the measurement and treatment of impulsivity in older adults.

\section{Developmental Considerations for the Measurement of Impulsivity in Older Adults}

Comparing impulsivity and its relationship with risk-taking behaviors across

developmental stages depends on valid measurement of the construct across the lifespan. Normal aging is associated with cognitive, affective, and sensorimotor changes (Samanez-Larkin \& Knutson, 2015; Samanez-Larkin et al., 2013) that could affect not only impulsivity in older adults, but also the type of impulsivity and the validity of the assessments used to measure impulsivity (Argyriou et al., 2018). For example, many behavioral impulsivity tasks rely on speed of processing, which slows with age, thus confounding the measurement of this tendency in older adults. Additionally, some personality-based scales ask about risk taking behaviors that 
require a certain amount of physical agility (e.g., skydiving, riding a motorcycle) that would be more difficult as one ages. Further complicating the matter is that most of these measures were originally developed and tested in young adult, college samples; thus, it is unclear whether they are valid and reliable in middle to older adults. To evaluate differences in impulsivity across the lifespan, we need self-report and behavioral task measures that can provide valid and reliable assessments of impulsivity across age groups. Only then will we be able to draw valid conclusions about group differences in impulsivity across age.

Some of this work has begun. A recent study (Argyriou et al., 2019) assessed the invariance of the UPPS-P Impulsive Behavior Scale across the adult lifespan (age range: 18-85 years old) in a community sample that closely matches the U.S. general population based on age, sex, race, immigrant status, language, education, and income. This measurement invariance analysis tested whether the UPPS-P scale can produce valid and reliable measurements of the impulsivity traits across age. If it does, then valid comparisons can be made across age groups; if it does not, then comparisons across the adult lifespan cannot be validly made using this scale as they can lead to results that inflate, deflate, or distort the appearance of real group differences. The findings of this study generally supported the invariance of this measure across young, middle, and older adulthood. However, three items with differential item functioning were identified that are less valid indicators for impulsivity traits in older ages (e.g., Item 51: 'I would like to go scuba diving'). The authors recommended removing these items for more valid comparisons of self-reported impulsivity across age (Argyriou et al., 2019). After removing these items, the authors found a small decrease in sensation seeking with age and there were no differences in the other traits across age. Previously reported large decreases in impulsivity with 
age may reflect less valid measurement of impulsivity among older adults using existing scales and measures.

Assessing the applicability of behavioral tasks across the adult lifespan is more complex than with self-report measures. Performance on a behavioral measure of impulsivity may be confounded with other processes that change with normal aging. For example, for prepotent response inhibition tasks, such as the Stop Task, slower reaction time, the most commonly used index of prepotent response inhibition, could reflect aging-related slower motoric dexterity or processing speed (Charlton et al., 2008; Sebastian et al., 2013), although the task takes into account individual differences in reaction time to some extent. Older individuals may be less familiar with computer technology (Iverson et al., 2009), which could reduce the validity of using these tasks in older age groups. Stereotype threat (i.e., the fear one may have that they may perpetuate or exemplify a negative stereotype about themselves) may further affect performance disproportionately among older adults (Mazerolle et al., 2012). Thus, task performance may be affected by other factors in addition to, or instead of, impaired ability to suppress automatic responses. In the case of delay discounting tasks, there may be age-related changes in the value of the rewards or in the perception of elapsed time of the delays. Rewards in delay discounting procedures may be real or hypothetical; cognitive control mechanisms are important for hypothetical rewards, whereas affective/motivation processes are important for real rewards. Changes in motivational goals and life experience with normal aging affect the subjective value of rewards and motivation to discount it (Samanez-Larkin et al., 2013), which may change the meaning of a reward and its discounting time frame. Any of these factors might threaten the validity of the measurement and complicate direct comparisons of performance across age (Argyriou et al., 2018). Addressing these confounds that disproportionately influence older 
adults will result in measures of impulsivity that are suitable for comparisons across the adult lifespan.

\section{Developmental Considerations for the Treatment of Impulsivity in Older Adults}

Several studies have examined the relationship between impulsivity and substance use treatment outcomes. A recent meta-analysis found that high negative urgency and lack of premeditation at intake to a substance use disorder treatment program were associated with worse treatment outcomes (Hershberger et al., 2017), supported by some work (Loree et al., 2015; Stevens et al., 2014), although not others (e.g., Tomko et al 2016). A recent review supported this idea and furthermore recommended the development of novel treatment methods to target other facets of impulsivity (Um et al., 2018).

Overall, there are few treatments specifically targeting impulsivity and fewer that have been tested, although some have made suggestions on possible treatment approaches for these tendencies. Zapolski et al. (2010) suggested that negative urgency might be effectively modified through distress tolerance strategies or emotion regulation approaches; sensation seeking may be modified through developing a bank of safe, stimulating activities that do not involve substance use; positive urgency may be modified through the teaching of adaptive techniques for savoring success and positive mood; lack of premeditation may be modified through cognitive meditation training; and lack of perseverance may be modified through stimulant medication and the use of goal setting and planning training. Tomko and colleagues (2016) suggested that contingency management may be a particularly effective treatment approach for those high in impulsivity.

Some researchers have developed treatments to reduce impulsivity in adolescent and young adult populations, including some with substance use problems. These studies have targeted mainly self-report, trait measures of impulsivity, which may be difficult to change. One 
study in a small sample of African American female college students found that training in emotion modulation decreased negative and positive urgency and reduced risk-taking (Weiss et al., 2015). Similarly, a school-based treatment based in Dialectical Behavioral Therapy reduced risk-taking behaviors in a small sample of high school adolescents (Zapolski et al., 2017). Interestingly, among adolescents in a smoking cessation program combining contingency management and cognitive behavioral therapy, lower impulsivity scores at treatment onset were associated with increased likelihood of successful abstinence (Krishnan-Sarin et al 2007).

Some approaches (e.g., distress tolerance or emotion regulation to modify negative urgency) appear to be easily generalizable to older adult populations. However, several aspects of impulsivity-targeted treatments designed for youth may not be applicable for middle-to-older adults. For example, school-based interventions (e.g., the treatment tested by Zapolski et al., 2017) would be difficult to modify for older adults, because the content may not generalize, the time involved is large (9-week session), and the school setting may not have a comparable counterpart. Older adults might be treated in a work setting, but the viability of this option is limited by stigma, availability, and the tradeoff between work time and the time spent in group. Additionally, it would be difficult to identify adults to refer to such a treatment. In contrast to school-based interventions, the emotion modulation approach supported by Weiss et al. (2015) may be better suited to adults, as the content is delivered in an one-hour session, which could be administered via more accessible strategies (e.g., webinar, online module, orientation meeting).

It is not clear whether reducing impulsivity would be effective in middle-to-older adults. Of note, the meta-analysis conducted by Hershberger et al. (2017) reviewed studies examining substance use disorder treatment effectiveness, not impulsivity in particular. These studies reported greater age ranges than typically included in impulsivity research, thus allowing for a 
better understanding of how treatment may affect and may be affected by impulsivity. Although Hershberger et al. (2017) did not examine age as a moderator of treatment effectiveness (due to the limited number of studies that met inclusion criteria), the effects reported (that negative urgency and lack of premeditation reduced treatment effectiveness) were found in treatment samples with a mean age of approximately 35 years of age (ranges from mid-twenties to midforties), indicating that, at least, the findings suggesting the influence of impulsivity on substance use disorder treatment outcomes likely apply to young-to-middle adults. They additionally reported significant, but very small, reductions in negative urgency and sensation seeking during treatment (Hershberger et al., 2017).

\section{Suggestions for Measurement and Treatment of Impulsivity in Older Adults}

We thus suggest that, although promising, existing measurements of and treatments for impulsivity may need to be modified extensively to maximize their effectiveness in middle-toolder adults. Previous attempts to modify psychological treatment with older adults have been proposed (e.g., Knight, Nordhus, \& Satre, 2003). We use the framework provided by Evans (2007) to suggest how to modify assessment and treatment of impulsivity for older adults, including addressing cognitive changes, addressing sensory impairment, incorporating physical health limitations, utilizing flexible settings and formats, and modifying content.

Addressing cognitive changes. Cognitive changes may confound behavioral measures of impulsivity in older adults (see review by Argyriou et al., 2018), reducing the valid measurement of these tendencies in this group. Additionally, many of the approaches suggested by Zapolski and colleagues (2010) utilize teaching and applying new skills (e.g., emotion regulation, distress tolerance, goal setting) through psychoeducation and practice. Impairments in memory may reduce the effectiveness of such approaches. Similar to what is suggested by Evans (2007), using 
memory training and aids, and ensuring the presentations of information in multiple formats can help increase the application of existing treatments to older populations. For instance, assistive devices, such as written handouts and online modules, may ease the stress of taking notes or recalling key components after intervention sessions (Foulk et al., 2014).

Addressing sensory impairment. Visual and hearing impairment can limit the validity of any self-report or behavioral measure of impulsivity and can also limit the effectiveness of any treatment training, sometimes without awareness. Assessing for such impairment prior to assessment and treatment planning is important so that modifications can be made to address any identified impairments (e.g., larger font, more contrast between stimuli and background during behavioral tasks, audio narration, etc.).

Incorporating physical health limitations. Physical limitations may limit self-report and behavioral measurement of impulsivity - for example, an older person may say "no" to the item "I would enjoy water skiing" because physical limitations would make the activity unpleasant, not because they are low in sensation seeking (see Argyriou et al., 2018). Additionally, treatments that aim to develop appropriate behavioral substitutions for impulsive behaviors will be somewhat limited by the physical health of the individual - for example, relaxation techniques may be difficult in those with many physical ailments or replacement behaviors may not all be possible for the individual. Additionally, physical ailments can cause serious stress, complicating treatment because of additional symptomology, pain management, and the impact of medical treatment and medication. Thus, we suggest including a measure of physical health, discussing limitations with the client, and including feedback from the participant as to the feasibility of homework assignments. 
Utilizing flexible settings and format. Settings and formats used for adolescent and younger adult populations often include school or university settings, increasing availability and feasibility of treatment. Middle-to-older adults likely have additional barriers with attending treatment including limited time and resources (e.g., childcare or eldercare responsibilities, long work hours, transportation limitations). Flexible settings for treatment (e.g., treatment in own home or in primary care settings, Evan, 2007; online or phone interventions) and reduced time commitments will likely increase access to care for these groups. Treatment sessions can also be shortened (i.e., from 60 minutes to 30 minutes) or incorporate breaks as needed. Additionally, some data suggest that group treatments may be more effective for older adults, as it provides peer support and opportunities to try new skills (Evans, 2007, Foulk et al 2014). Using this intervention format may also lower financial barriers since it is covered by Medicare and most private insurance.

Modifying content. Some of the measurement or therapy content for impulsivity may not generalize well to older adults. For example, what impulsivity is in older adults may look quite different than in younger adults - with less reliance on traditional risk taking (e.g., sky diving, bungee jumping) and more reliance on behaviors still available to an aging population (e.g., unprotected sex, investments) - which may make traditional impulsivity measures less valid in older adults. Additionally, there is often a view that people are "too old to change," both by the therapist and the client, that may need to be challenged early on in treatment for it to be most effective (Evans, 2007). Evans (2007) also reviews other important considerations for treatment with older adults including ageist assumptions, stigma, and prejudice against younger therapists, all of which would need to be targeted in treatment.

\section{Conclusions}


In conclusion, extensive literature supports impulsivity as a significant risk factor in substance use. However, more needs to be done to fully extend this across the lifespan, particularly in how best to modify assessment and treatment of impulsivity in older adults. Because of the varied time-course of substance use, we propose that impulsivity research should move away from a predominant focus on adolescence and young adulthood to also include data on middle-to-older adulthood as an important time of transition to disordered use. Over-reliance on adolescents and young adults has likely led to an underappreciation of the role impulsivity may play among middle-to-older adults. Additionally, although some evidence suggests that selfreport impulsivity measures can validly assess impulsivity across the adult lifespan (e.g., Argyriou et al., 2019), researchers may want to rethink how we use behavioral tasks to assess impulsivity in these older groups, as performance is likely confounded with aging-related effects. Valid comparisons across the lifespan cannot be made until it is documented that the measures used to assess impulsivity produce valid and reliable data across age. Applying treatments designed to modify impulsivity to older adults requires consideration of important modifications in order to increase usefulness and effectiveness. We hope to catalyze work with impulsivity in middle-to-older adults. If impulsivity is fundamentally different in older adults or differentially impacts substance use or treatment outcomes, we require age specific strategies for identification, prevention, and treatment to effectively reduce risks in this clinically relevant group. 


\section{References}

Aragues, M., Jurado, R., Quinto, R., \& Rubio, G. (2011). Laboratory paradigms of impulsivity and alcohol dependence: A review. European Addiction Research. https://doi.org/10.1159/000321345

Argyriou, E., Um, M., Carron, C., \& Cyders, M. (2018). Age and impulsive behavior in drug addiction: A review of past research and future directions. Pharmacology, Biochemistry, and Behavior, 164, 106-117. doi:10.1016/j.pbb.2017.07.013

Argyriou, E., Um, M., Wu, W., \& Cyders, M. A. (2019). Measurement Invariance of the UPPS-P Impulsive Behavior Scale Across Age and Sex Across the Adult Life Span. Assessment, https://doi.org/10.1177/1073191119832660.

Blakemore, S. J., \& Robbins, T. W. (2012). Decision-making in the adolescent brain. Nature Neuroscience. https://doi.org/10.1038/nn.3177

Breslow, R. A., Castle, I.-J. P., Chen, C. M., \& Graubard, B. I. (2017). Trends in alcohol consumption among older americans: National health interview surveys, 1997 to 2014. Alcoholism: Clinical and Experiemental Research, 41, 976-986.

Casey, B. J., Jones, R. M., \& Hare, T. A. (2008). The adolescent brain. Annals of the New York Academy of Sciences. https://doi.org/10.1196/annals.1440.010

Charlton, R. A., Landau, S., Schiavone, F., Barrick, T. R., Clark, C. A., Markus, H. S., \& Morris, R. G. (2008). A structural equation modeling investigation of age-related variance in executive function and DTI measured white matter damage. Neurobiology of aging, 29(10), 1547-1555.

Collado, A., Felton, J.W., Macpherson, L., Lejuez, C.W. (2014). Longitudinal trajectories of sensation seeking, risk taking propensity, and impulsive behavior across early to middle adolescence. Addict. Behav. 39, 1580-1588. http://dx.doi.org/10.1016/j.addbeh.2014.01.024.

Coskunpinar, A., Dir, A. L., \& Cyders, M. A. (2013). Multidimensionality in impulsivity and alcohol use: A meta-analysis using the UPPS model of impulsivity. Alcoholism: Clinical and Experimental Research, 37(9), 1441-1450. https://doi.org/10.1111/acer.12131

Crone, E. A., \& Dahl, R. E. (2012). Understanding adolescence as a period of social-affective engagement and goal flexibility. Nature Reviews Neuroscience. https://doi.org/10.1038/nrn3313

Cyders, M. A., \& Coskunpinar, A. (2011). Measurement of constructs using self-report and behavioral lab tasks: Is there overlap in nomothetic span and construct representation for impulsivity? Clinical Psychology Review, 31(6), 965-982. https://doi.org/10.1016/j.cpr.2011.06.001

Cyders, M. A., \& Smith, G. T. (2007). Mood-based rash action and its components: positive and negative urgency. Personality and Individual Differences, 43, 839-850.

Cyders, M. A., Smith, G. T., Spillane, N. S., Fischer, S., Annus, A. M., \& Peterson, C. (2007). Integration of impulsivity and positive mood to predict risky behavior: Development and validation of a measure of positive urgency. Psychological Assessment, 19(1), 107-118. https://doi.org/10.1037/1040-3590.19.1.107

Dick, D. M., Smith, G., Olausson, P., Mitchell, S. H., Leeman, R. F., O’Malley, S. S., \& Sher, K. (2010). Understanding the construct of impulsivity and its relationship to alcohol use disorders. Addiction Biology. https://doi.org/10.1111/j.1369-1600.2009.00190.x

Evans, C. (2007). Cognitive-behavioural therapy with older people. Advances in Psychiatric Treatment, 13(2), 111-118. 
Ernst, M., \& Fudge, J. L. (2009). A developmental neurobiological model of motivated behavior: anatomy, connectivity and ontogeny of the triadic nodes. Neuroscience \& Biobehavioral Reviews, 33(3), 367-382.

Evenden, J. L. (1999). Varieties of impulsivity. Psychopharmacology. https://doi.org/10.1007/PL00005481

Fineberg, N. A., Chamberlain, S. R., Goudriaan, A. E., Stein, D. J., Vanderschuren, L. J. M. J., Gillan, C. M., ... Potenza, M. N. (2014). New developments in human neurocognition: Clinical, genetic, and brain imaging correlates of impulsivity and compulsivity. CNS Spectrums.

Foulk, M. A., Ingersoll-Dayton, B., Kavanagh, J., Robinson, E., \& Kales, H. C. (2014). Mindfulness-based cognitive therapy with older adults: an exploratory study. Journal of gerontological social work, 57(5), 498-520.

Galván, A. (2010). Adolescent development of the reward system. Frontiers in human neuroscience, 4,6 .

Gerard Moeller, F., \& Dougherty, D. M. (2002). Impulsivity and substance abuse: What is the connection? Addictive Disorders and Their Treatment. https://doi.org/10.1097/00132576200205000-00002

Green, L., Fry, A. F., \& Myerson, J. (1994). Discounting of delayed rewards: A life-span comparison. Psychological science, 5(1), 33-36.

Harden, K. P., \& Tucker-Drob, E. M. (2011). Individual differences in the development of sensation seeking and impulsivity during adolescence: further evidence for a dual systems model. Developmental psychology, 47(3), 739.

Hershberger, A. R., Um, M., \& Cyders, M. A. (2017). The relationship between the UPPS-P impulsive personality traits and substance use psychotherapy outcomes: a meta-analysis. Drug and alcohol dependence, 178, 408-416.

Heyman, G. M. (2013). Quitting Drugs: Quantitative and Qualitative Features. Annual Review of Clinical Psychology. https://doi.org/10.1146/annurev-clinpsy-032511-143041

Iverson, G. L., Brooks, B. L., Ashton, V. L., Johnson, L. G., \& Gualtieri, C. T. (2009). Does familiarity with computers affect computerized neuropsychological test performance?. Journal of Clinical and Experimental Neuropsychology, 31(5), 594-604.

Jaeger, A. (2013). Inhibitory control and the adolescent brain: a review of fMRI research. Psychology \& Neuroscience, 6(1), 23-30.

Knight, B. G., Nordhus, I. H., \& Satre, D. D. (2003). Psychotherapy with older adults. Handbook of Psychology, 453-468.

Koob, G. F. (2004). Allostatic view of motivation: implications for psychopathology. Nebraska Symposium on Motivation. Nebraska Symposium on Motivation.

Koob, G. F., \& Le Moal, M. (2008). Addiction and the Brain Antireward System. Annual Review of Psychology, 59(1), 29-53. https://doi.org/10.1146/annurev.psych.59.103006.093548

Krishnan-Sarin, S., Reynolds, B., Duhig, A. M., Smith, A., Liss, T., McFetridge, A., ... \& Potenza, M. N. (2007). Behavioral impulsivity predicts treatment outcome in a smoking cessation program for adolescent smokers. Drug and alcohol dependence, 88(1), 79-82.

Littlefield, A. K., \& Sher, K. J. (2015). Alcohol Use Disorders in Young Adulthood. In Young Adult Mental Health. https://doi.org/10.1093/med:psych/9780195332711.003.0018 
Littlefield, A. K., Sher, K. J., \& Wood, P. K. (2009). Is \&quot;maturing out\&quot; of problematic alcohol involvement related to personality change? Journal of Abnormal Psychology, 118(2), 360-374. https://doi.org/10.1037/a0015125

Littlefield, A. K., \& Sher, K. J. (2016). 10 personality and substance use disorders. The Oxford Handbook of Substance Use and Substance Use Disorders: Two-Volume Set, 351.

Littlefield, A. K., Stevens, A. K., Ellingson, J. M., King, K. M., \& Jackson, K. M. (2016). Changes in negative urgency, positive urgency, and sensation seeking across adolescence. Personality and individual differences, 90, 332-337.

López-Caneda, E., Rodríguez Holguín, S., Cadaveira, F., Corral, M., \& Doallo, S. (2013). Impact of alcohol use on inhibitory control (and vice versa) during adolescence and young adulthood: a review. Alcohol and alcoholism, 49(2), 173-181.

Loree, A. M., Lundahl, L. H., \& Ledgerwood, D. M. (2015). Impulsivity as a predictor of treatment outcome in substance use disorders: Review and synthesis. Drug and alcohol review, 34(2), 119-134.

Lynam, D. R., Smith, G. T., Whiteside, S. P., \& Cyders, M. A. (2006). The UPPS-P: Assessing five personality pathways to impulsive behavior. West Lafayette, IN: Purdue University.

Mazerolle, M., Régner, I., Morisset, P., Rigalleau, F., \& Huguet, P. (2012). Stereotype threat strengthens automatic recall and undermines controlled processes in older adults. Psychological science, 23(7), 723-727.

Olson, E. A., Hooper, C. J., Collins, P., \& Luciana, M. (2007). Adolescents' performance on delay and probability discounting tasks: contributions of age, intelligence, executive functioning, and self-reported externalizing behavior. Personality and individual differences, 43(7), 1886-1897.

Pedersen, S. L., Molina, B. S., Belendiuk, K. A., \& Donovan, J. E. (2012). Racial differences in the development of impulsivity and sensation seeking from childhood into adolescence and their relation to alcohol use. Alcoholism: Clinical and Experimental Research, 36(10), 1794-1802.

Romer, D., \& Hennessy, M. (2007). A biosocial-affect model of adolescent sensation seeking: The role of affect evaluation and peer-group influence in adolescent drug use. Prevention Science, 8(2), 89.

Samanez-Larkin, G. R., \& Knutson, B. (2015). Decision making in the ageing brain: changes in affective and motivational circuits. Nature Reviews Neuroscience, 16(5), 278.

Samanez-Larkin, G. R., Li, S. C., \& Ridderinkhof, K. R. (2013). Complementary approaches to the study of decision making across the adult life span. Frontiers in neuroscience, 7, 243.

Sebastian, A., Baldermann, C., Feige, B., Katzev, M., Scheller, E., Hellwig, B., ... \& Klöppel, S. (2013). Differential effects of age on subcomponents of response inhibition. Neurobiology of aging, 34(9), 2183-2193.

Sharma, L., Markon, K. E., \& Clark, L. A. (2014). Toward a theory of distinct types of "impulsive" behaviors: A meta-analysis of self-report and behavioral measures. Psychological Bulletin. https://doi.org/10.1037/a0034418

Smith, G. T., Fischer, S., \& Fister, S. M. (2003). Incremental Validity Principles in Test Construction. Psychological Assessment. https://doi.org/10.1037/1040-3590.15.4.467

Smith, G. T., Fischer, S., Cyders, M. A., Annus, A. M., Spillane, N. S., \& McCarthy, D. M. (2007). On the validity and utility of discriminating among impulsivity-like traits. Assessment, 14, 155-170. https://doi.org/10.1111/1744-7941.12061 
Somerville, L. H., Jones, R. M., \& Casey, B. J. (2010). A time of change: behavioral and neural correlates of adolescent sensitivity to appetitive and aversive environmental cues. Brain and cognition, 72(1), 124-133.

Stautz, K., \& Cooper, A. (2013). Impulsivity-related personality traits and adolescent alcohol use: A meta-analytic review. Clinical Psychology Review, 33(4), 574-592. https://doi.org/10.1016/j.cpr.2013.03.003

Steinberg, L. (2008). A social neuroscience perspective on adolescent risk-taking. Developmental review, 28(1), 78-106.

Steinberg, L., Albert, D., Cauffman, E., Banich, M., Graham, S., \& Woolard, J. (2008). Age differences in sensation seeking and impulsivity as indexed by behavior and self-report: evidence for a dual systems model. Developmental psychology, 44(6), 1764.

Stevens, L., Verdejo-García, A., Goudriaan, A. E., Roeyers, H., Dom, G., \& Vanderplasschen, W. (2014). Impulsivity as a vulnerability factor for poor addiction treatment outcomes: a review of neurocognitive findings among individuals with substance use disorders. Journal of Substance Abuse Treatment, 47(1), 58-72.

Tomko, R. L., Bountress, K. E., \& Gray, K. M. (2016). Personalizing substance use treatment based on pre-treatment impulsivity and sensation seeking: A review. Drug and alcohol dependence, 167, 1-7.

Um, M., Hershberger, A. R., Whitt, Z. T., \& Cyders, M. A. (2018). Recommendations for applying a multi-dimensional model of impulsive personality to diagnosis and treatment. Borderline Personality Disorder and Emotion Dysregulation. https://doi.org/10.1186/s40479-018-0084-X

Um, M., Hershberger, A. R., Whitt, Z. T., \& Cyders, M. A. (2018). Recommendations for applying a multi-dimensional model of impulsive personality to diagnosis and treatment. Borderline personality disorder and emotion dysregulation, 5(1), 6 .

VanderVeen, J. D., Hershberger, A. R., \& Cyders, M. A. (2016). UPPS-P model impulsivity and marijuana use behaviors in adolescents: A meta-analysis. Drug and Alcohol Dependence. https://doi.org/10.1016/j.drugalcdep.2016.09.016

Verdejo-García, A., Lawrence, A. J., \& Clark, L. (2008). Impulsivity as a vulnerability marker for substance-use disorders: Review of findings from high-risk research, problem gamblers and genetic association studies. Neuroscience and Biobehavioral Reviews. https://doi.org/10.1016/j.neubiorev.2007.11.003

Vergés, A., Jackson, K. M., Bucholz, K. K., Grant, J. D., Trull, T. J., Wood, P. K., \& Sher, K. J. (2012). Deconstructing the age-prevalence curve of alcohol dependence: Why "maturing out" is only a small piece of the puzzle. Journal of abnormal psychology, 121(2), 511.

Weiss, N. H., Tull, M. T., Davis, L. T., Searcy, J., Williams, I., \& Gratz, K. L. (2015). A preliminary experimental investigation of emotion dysregulation and impulsivity in risky behaviours. Behaviour Change, 32(2), 127-142.

Whiteside, S. P., \& Lynam, D. R. (2001). The five factor model and impulsivity: Using a structural model of personality to understand impulsivity. Personality and Individual Differences, 30(4), 669-689. https://doi.org/10.1016/S0191-8869(00)00064-7

Young, S. E., Corley, R. P., Stallings, M. C., Rhee, S. H., Crowley, T. J., \& Hewitt, J. K. (2002). Substance use, abuse and dependence in adolescence: Prevalence, symptom profiles and correlates. Drug and Alcohol Dependence. https://doi.org/10.1016/S03768716(02)00225-9 
Zapolski, T. C., \& Smith, G. T. (2017). Pilot study: Implementing a brief DBT skills program in schools to reduce health risk behaviors among early adolescents. The Journal of School Nursing, 33(3), 198-204.

Zapolski, T. C., Settles, R. E., Cyders, M. A., \& Smith, G. T. (2010). Borderline personality disorder, bulimia nervosa, antisocial personality disorder, ADHD, substance use: common threads, common treatment needs, and the nature of impulsivity. Independent Practitioner (Lutterworth, England), 30(1), 20.

Zorrilla, E. P., \& Koob, G. F. (2019). Impulsivity derived from the dark side: Neurocircuits that contribute to negative urgency. Frontiers in Behavioral Neuroscience.

https://doi.org/10.3389/fnbeh.2019.00136 